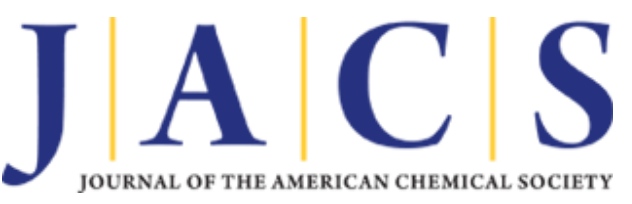

\title{
Article
}

\section{Few-layer Antimonene: Large Yield Synthesis, Exact Atomical Structure and Outstanding Optical Limiting}

Chengxue Huo, Xingming Sun, Zhong Yan, Xiufeng Song, Shengli Zhang, Zheng Xie, Ji-Zi Liu, Jianping Ji, Lianfu Jiang, Shuyun Zhou, and Haibo Zeng

J. Am. Chem. Soc., Just Accepted Manuscript • DOI: 10.1021/jacs.6b08698 • Publication Date (Web): 11 Oct 2016

Downloaded from http://pubs.acs.org on February 23, 2017

\section{Just Accepted}

"Just Accepted" manuscripts have been peer-reviewed and accepted for publication. They are posted online prior to technical editing, formatting for publication and author proofing. The American Chemical Society provides "Just Accepted" as a free service to the research community to expedite the dissemination of scientific material as soon as possible after acceptance. "Just Accepted" manuscripts appear in full in PDF format accompanied by an HTML abstract. "Just Accepted" manuscripts have been fully peer reviewed, but should not be considered the official version of record. They are accessible to all readers and citable by the Digital Object Identifier (DOI®). "Just Accepted" is an optional service offered to authors. Therefore, the "Just Accepted" Web site may not include all articles that will be published in the journal. After a manuscript is technically edited and formatted, it will be removed from the "Just Accepted" Web site and published as an ASAP article. Note that technical editing may introduce minor changes to the manuscript text and/or graphics which could affect content, and all legal disclaimers and ethical guidelines that apply to the journal pertain. ACS cannot be held responsible for errors or consequences arising from the use of information contained in these "Just Accepted" manuscripts.

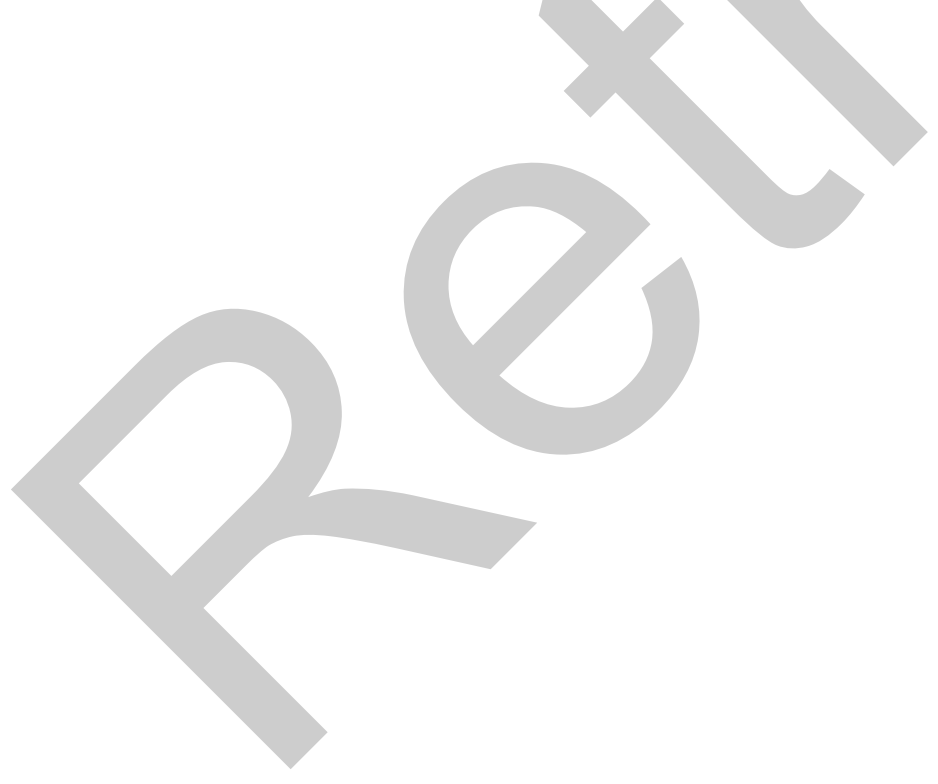


Few-layer Antimonene: Large Yield Synthesis, Exact Atomical Structure and Outstanding Optical Limiting

$$
\begin{aligned}
& \text { Chengxue Huo }{ }^{\# 1} \text {, Xingming Sun }{ }^{\# 2} \text {, Zhong Yan }{ }^{\# 1,3} \text {, Xiufeng Song }{ }^{\# 1,3} \text {, Shengli Zhang }{ }^{\# 1,3} \text {, Zheng } \\
& \text { Xie }^{* 2} \text {, Jizi Liu }{ }^{3} \text {, Jianping Ji }{ }^{1} \text {, Lianfu Jiang }{ }^{1} \text {, Shuyun Zhou }{ }^{2} \text {, Haibo Zeng }{ }^{* 1,3}
\end{aligned}
$$

${ }^{1}$ Institute of Optoelectronics \& Nanomaterials, College of Materials Science and Engineering, Nanjing University of Science and Technology, Nanjing 210094, China

${ }^{2}$ Key Laboratory of Photochemical Conversion and Optoelectronic Materials, Technical Institute of Physics and Chemistry, Chinese Academy of Sciences, Beijing 100190, China

${ }^{3}$ Herbert Gleiter Institute of Nanoscience, Nanjing University of Science and Technology, Nanjing 210094, China

\# These authors equally contribute to this work.

*Authors to whom correspondence should be addressed, e-mail: zeng.haibo@njust.edu.cn, zhengxie@mail.ipc.ac.cn. 
Abstract: The predictions of arsenene and antimonene open a gate to new two-dimensional (2D) materials. In contrast to the severe unstability of black phosphorus in ambient atmosphere, arsenene and antimonene were recently predicted to be of high stability, as well as outstanding physical and chemical properties, such as extremely high mobility, superior thermal conductivity, high refractive index, and directionally optically transparent. Significantly, monolayer and few-layer antimonenes were recently experimentally fabricated by mechanical exfoliation, however the yield was very low and there's lack of clear characterizations on the atomical structure and potential applications, which are critical and necessary to promote the developments of this field. Here, we report on a high-yield experimental preparation of high quality, few-layer antimonenes via liquid exfoliation, their atomic level structural elucidation, and unexpected but outstanding nonlinear optical limiting properties even better than graphene in the visible and near infrared region $(532 \mathrm{~nm}-2000 \mathrm{~nm}$ ) and high transmission (more than $80 \%$ ) when dispersed in solutions or high concentration doped in Ormosil gel glasses, which might lead to many promising applications in nonlinear optical fields such as laser protection. This work demonstrates that Group 15 2D materials beyond BP could be not only a new 2D crystal family with stability in ambient condition, but also of unique properties and applications.

Key words: two-dimensional materials; arsenene; antimonene; optical limiting 


\section{Introduction}

Recently, the rediscovery of black phosphorus (BP) as a two-dimensional (2D) layered material has expanded the 2D family into Group 15 (the Nitrogen Group) elements. ${ }^{1-6}$ The unique properties of BP, such as the thickness dependent electronic bandgap $(0.3 \mathrm{eV} \sim$ ca. $2 \mathrm{eV}),{ }^{5}$ high carrier mobility (up to $1000 \mathrm{~cm}^{2} \mathrm{~V}^{-1} \mathrm{~s}^{-1}$ at room temperature), ${ }^{6}$ and anisotropic in-plane properties, ${ }^{2}$ endow BP promising applications in nanoelectronic and optoelectronic. ${ }^{3}$ However, atomically thin BP is unstable in air, it is rather challenging to handle in laboratories, let alone industrial applications. ${ }^{8-9}$

Fortunately, other 2D Group 15 materials such as arsenene and antimonene, i.e. 2D arsenic (As) and antimony ( $\mathrm{Sb}$ ) with few-layers, might possess high stabilities according to our recent theoretical study. ${ }^{10}$ Interestingly, though bulk As and Sb are typical semimetals, they transform into semiconductors with band gaps of $2.49 \mathrm{eV}$ and $2.28 \mathrm{eV}$ when thinned to one atomic layer, which just fills the bandgap vacancy among existing 2D semiconductors, such as BP, $\mathrm{MoS}_{2}$ (below $2 \mathrm{eV}$ ) and $\mathrm{BN}$ (above 5 $\mathrm{eV}){ }^{10}$ Since then, intensive theoretical efforts have been devoted to arsenene and antimonene. ${ }^{11-13}$ Among others, recent studies predicted that arsenene and antimonene could have diverse allotropes and interesting properties, such as extremely high mobility, ${ }^{14}$ superior thermal conductivity, ${ }^{15}$ promising spintronic properties, ${ }^{16}$ and wonderful optical properties like high refractive index, ${ }^{17}$ wide range absorption, ${ }^{18}$ directionally optically transparent. ${ }^{18}$

Despite these theoretical achievements, there are only a few experimental studies on arsenene and antimonene. ${ }^{19-20}$ Recently, Tsai et al. successfully synthesized multilayer As nanoribbons on InAs substrate using the plasma-assisted process and the estimated $\sim 2.3 \mathrm{eV}$ band gap indicates the potentials for $2 \mathrm{D}$ optoelectronic devices. ${ }^{19}$ However, the obtained multilayer As nanosheet was $14 \mathrm{~nm}$ thick and strongly confined by InN epitaxial substrate. Lei et al used molecular beam epitaxy (MBE) method grown few-layer antimonene on $\mathrm{Sb}_{2} \mathrm{Te}_{3}$ surfaces, which shows few-layer antimonene can be existed. ${ }^{20}$ However, there's lack of a detailed characterization and potential applications, which are important and necessary to 
promote the developments of this field. Thus, for the emerging research hotspots of arsenene and antimonene, it is very urgent to develop a facile and high yield preparation method to achieve high quality and large quantity antimonene, accurately elucidate their atomical structure, and explore their unique properties.

Here, we demonstrate that high-quality, few-layer antimonene with high stability can be produced in large quantities via liquid exfoliation in ambient conditions, especially present their accurate atomical structure, and report their unexpected but outstanding nonlinear optical (NLO) and optical limiting (OL) properties. X-ray diffraction (XRD), Raman spectroscopy and transmission electron microscopy (TEM) reveal that the obtained few-layer antimonenes have layered atomic structure consisting of buckled hexagonal rings as in the $\beta$-phase bulk antimony. To our surprise, few-layer antimonene exhibits wonderful OL properties in the visible and near infrared ray (NIR) region $(532 \mathrm{~nm} \sim 2000 \mathrm{~nm})$, better than all the reported 2D materials including graphene. Over $90 \%$ energy can be limited for few-layer antomonene dispersion at $532 \mathrm{~nm}$, which is better than graphene in the same condition. And few-layer antimonene can also be high concentration doped in the organically modified silicate (Ormosil) gel glasses, which might lead to many promising applications in NLO fields such as laser protection.

\section{Results and Discussion}

Large-scale Preparation of Few-layer Antimonene. Antimony is a layered material with weak interlayer van der Waals interactions, but strong interlayer covalent bonds. Liquid phase sonication has been proven effective in breaking such weak van der Waals forces, and hence can exfoliate layered materials into atomically thin sheets in large quantities. ${ }^{21-27}$ Figure 1 shows the schematic diagram of sonication liquid exfoliation. By sonication, the solvent molecules enter into and expand the interlamination spaces, and finally clave the layers of bulk materials into single- and few-layer nanosheets. According to Coleman' work, ${ }^{21,23}$ if the surface energy factors between solvents and layered materials match well, the energy cost of the exfoliation 
would be minimized. Since the surface energy of Sb nanosheet is unknown, we tried a wide range of solvents with different surface tension from deionized water to ethanol, Table S1 gives the information about solvents we tried. Our experiments proved that ethanol is a good choice for the exfoliation of antimony. The details of sample preparation are given in Methods and Supporting Information.

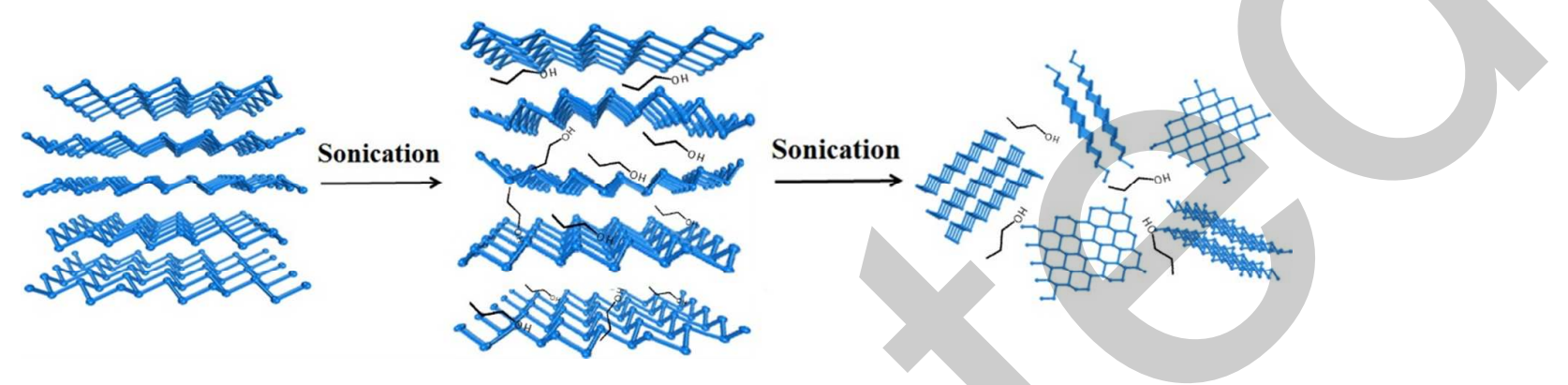

Figure 1. The schematic diagrams of sonication liquid exfoliation. By sonication, the molecules of solution enter into the layered structure of $\mathrm{Sb}$ and then the layers can be exfoliated.

Unlike graphene, monolayer antimonene consists of buckled structure of interlinked ruffled hexagonal rings (Figure 2a). This is the crystal structure of $\beta$-phase antimony based on the hexagonal coordinate system. Though antimony belongs to rhombohedral system, hexagonal coordinate system is convenient to describe its structure. And the detailed explanations about the two crystal systems are given in Supporting Information (Figure S1). Note that as the starting material, Sb powders have obviously stacked layered structure, which can be visualized in the scanning electron microscope (SEM) image (Figure 2b). We can see the cleavage planes and cleavage steps clearly in the image, indicating weaker binding forces among layers than those of in-plane binding. In fact, these match well with the theoretical calculation, ${ }^{10}$ which shows the layer-to-layer interaction inside $\mathrm{Sb}$ is van der Waals interactions. The typical intermediate state in Figure $2 \mathrm{c}$ verifies the preferential exfoliation along cleavage planes, but not perpendicular to them, which determines the final products as ultrathin sheets but not nanoparticles. Such preferential cutting was also confirmed by a typical atomic force microscopy (AFM) image as shown in 
Figure 1d, where two ultrathin sheets are separating and the synchronous fragmentation is very slight. And there's an obvious terraces in the blue circle. The preferential exfoliation along cleavage planes is also proven by XRD (Figure S2). Compared to the bulk powder, the (003) and (006) diffraction peaks almost disappeared after exfoliation, which confirms the preferential exfoliation along cleavage planes induced by sonication in such conditions.

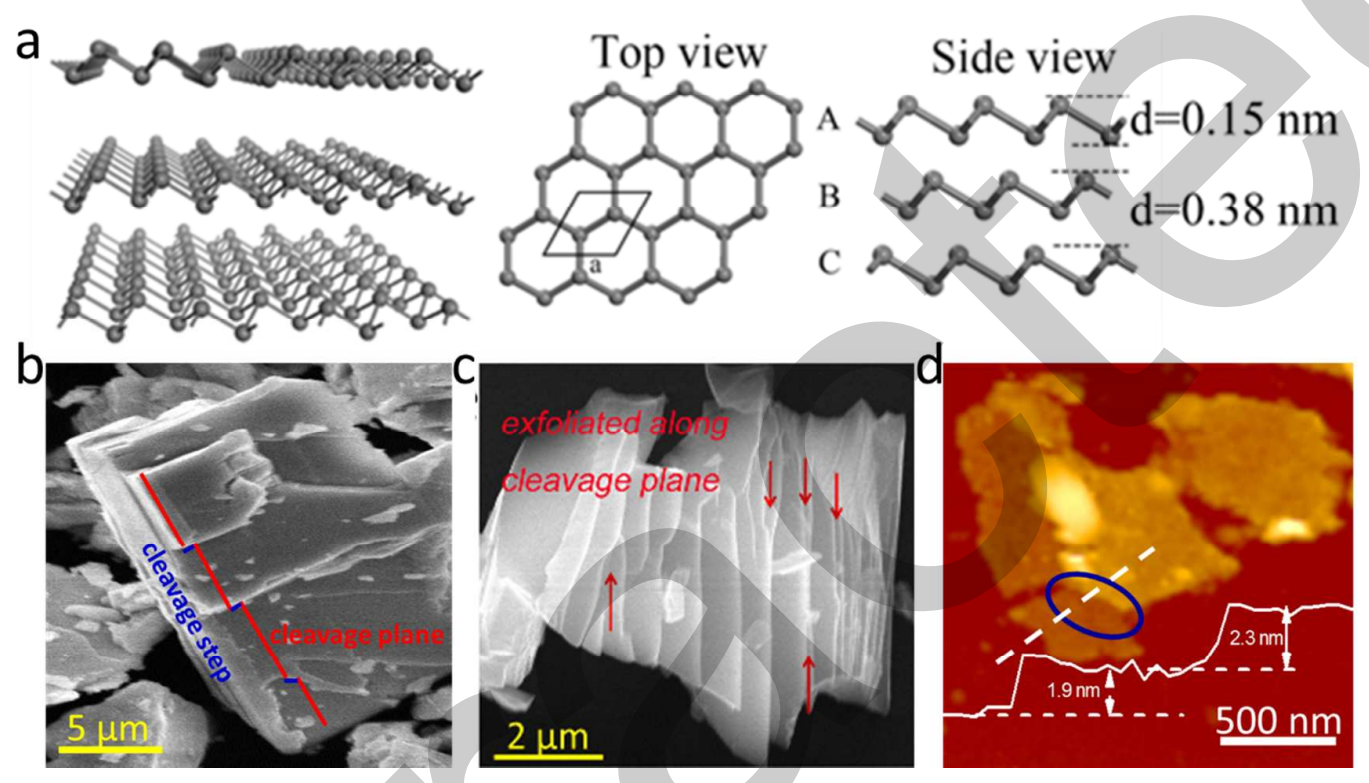

Figure 2. (a) Crystal structure of $\beta$-phase antimony. (b) SEM image of layered antimony powders, the cleavage planes and cleavage step can be seen clearly. (c) SEM image of layered antimony powders during the exfoliation, the layers were exfoliated along cleavage plane. (d) AFM image of Sb nanosheets during the exfoliation, the cleavage step can be seen (blue cycle) and the height of the cleavage step can be calculated by measuring the thickness of cleavage planes.

High Stability of Few-layer Antimonene Colloid. After sonication and centrifugation, the formed few-layer antimonene colloids are very stable, even after 30 days, which evidenced by the obvious Tyndall effect as shown in the Figure $3 \mathrm{a}$ and time-dependent absorption spectra (Figure 3b). As we can see, there's no obvious change as time increased, which prove the colloid is stable in ambient condition.

All the antimonene colloids show wide broadband (300-2500 nm) light 
absorption ability (Figure 3c). The dependence of the normalized absorbance intensity $(\mathrm{A} / \mathrm{L})$ at $1500 \mathrm{~nm}$ on few-layer antimonene concentration (Figure $3 \mathrm{c}$ shows the concentration-dependent absorption spectra of few-layer antimonene colloids) was plotted in Figure 3d, exhibiting very well linear relationship of $\mathrm{A} / \mathrm{L}=\alpha \mathrm{C}$ ( $\alpha$, the extinction coefficient), which is in agreement with Lambert-Beer behavior. The obtained calculated $\alpha$ value is $166 \mathrm{mLmg}^{-1} \mathrm{~m}^{-1}$, which suggests that the few-layer antimonenes can be well dispersed in such solvent. We can get a stable colloid with concentrations up to $2.5 \mathrm{mg} / \mathrm{mL}$. Such effective exfoliation fabrication proves that the predicted antimonene can really exist at a free standing state in ambient condition, and the large quantity and good dispersity will endow them many unique application potentials.
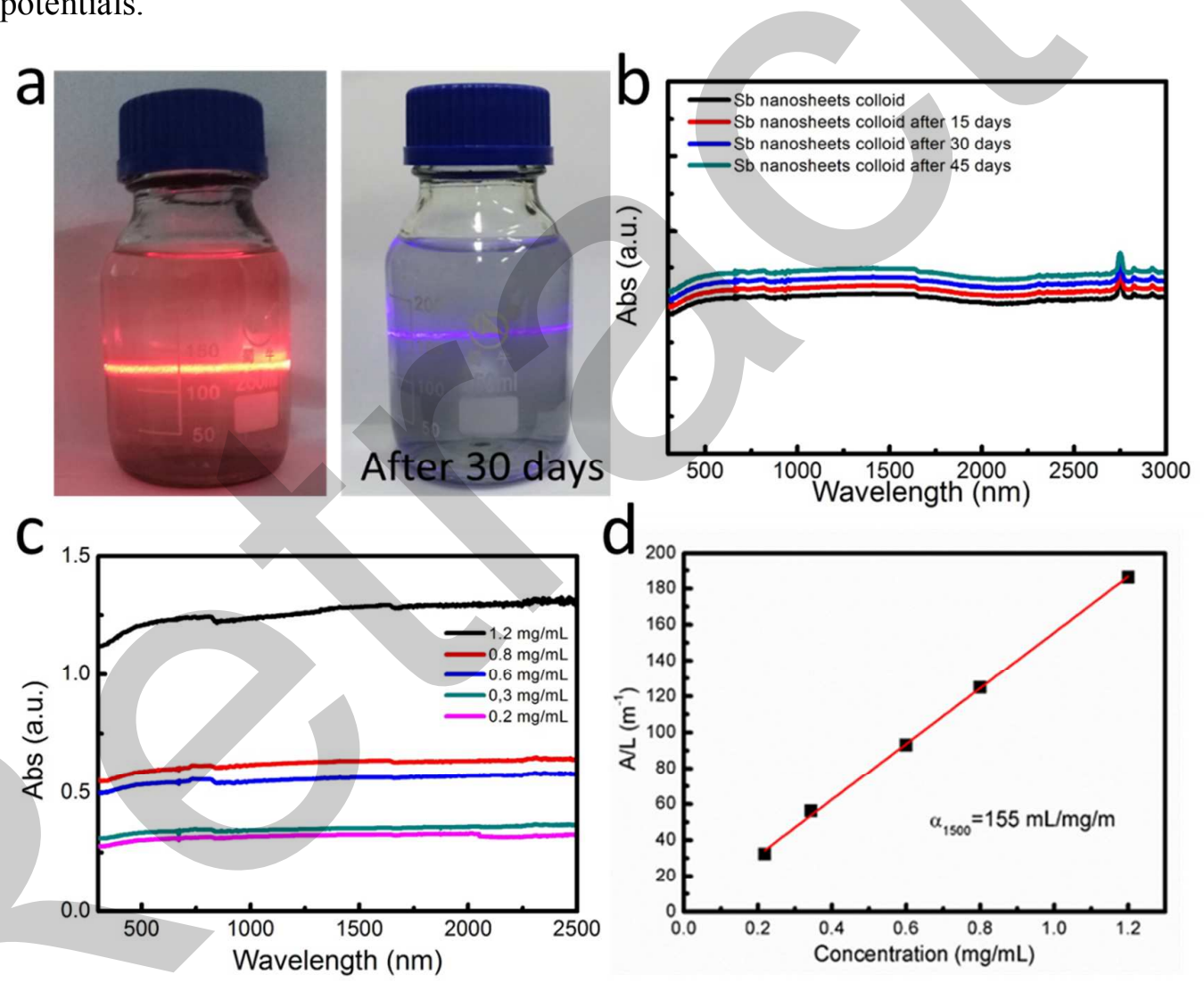

Figure 3. (a) Image of few-layer antimonene colloid with Tyndall effect, the left one is the image of colloid just prepared and the right one shows the same colloid after 30 days. (b) The time-dependent UV-Vis, which confirms the stability of few-layer antimonene colloid. (c) The concentration-dependent absorption spectra of few-layer antimonene colloids. (d) Normalized absorbance intensity over characteristic length of 
the cell (A/L) at different concentrations for $\lambda=1500 \mathrm{~nm}$.

Characterization of Few-layer Antimonene. Compared with other methods, sonication exfoliation is very effective to obtain $2 \mathrm{D}$ materials with very large quantity. We dispersed flakes of the above sample for further characterizations making use of coffee-ring effect (Figure 4a). ${ }^{28-29}$ By this way, we can get isolated nanosheets as higher-magnification SEM image (Figure 4b) showed in the edge of the substrate, and we can also see the few-layer antimonene packed by randomly oriented in the central area of the coffee rings. The statistical analysis over one hundred nanosheets showed that the lateral sizes obey Gaussian distribution with the average size of $600 \mathrm{~nm}$ (Figure 4d).
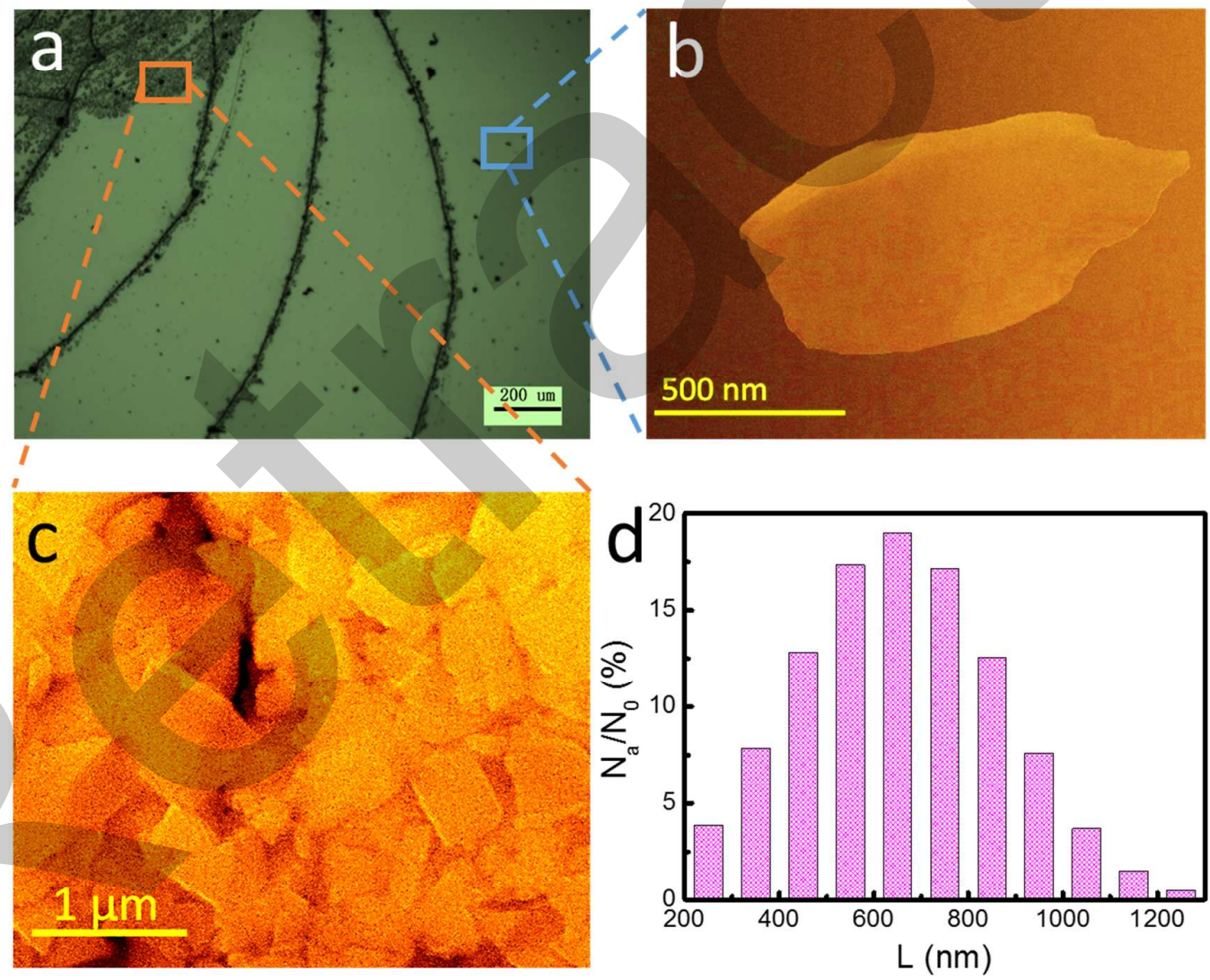

Figure 4. (a) Optical image of deposited nanosheets on $\mathrm{SiO}_{2} / \mathrm{Si}$ substrate, showing the "coffee-ring" structure. (b) SEM image of individual nanosheet located at the outer region of coffee rings. (c) SEM image of the central area of the rings. (d) The 
statistical diagram of nanosheets lateral size on 100 flakes.

To obtain the information about thickness of the $\mathrm{Sb}$ nanosheets, AFM measurements are necessary. Figure 5a gave the AFM image of dispersive nanosheets with height analysis. From statistics of 100 flakes, we found that most flakes' thicknesses were below $15 \mathrm{~nm}$ and almost $50 \%$ of the flakes were thinner than $10 \mathrm{~nm}$ (Figure 5b).
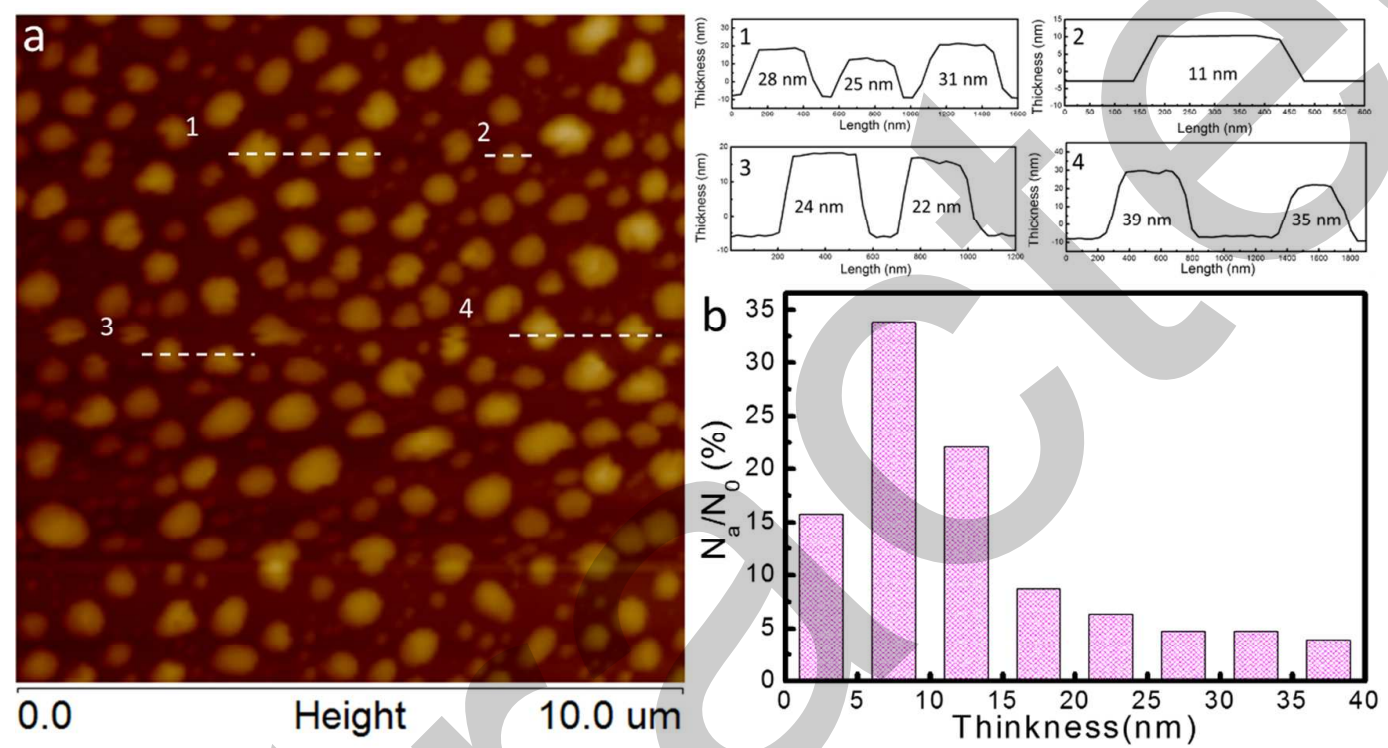

Figure 5. (a) AFM image of deposited $\mathrm{Sb}$ nanosheets on $\mathrm{SiO}_{2} / \mathrm{Si}$ substrate and the height analysis of typical samples. (b) Statistical histogram of the thickness on 100 nanosheets, almost $50 \%$ of the flakes were thinner than $10 \mathrm{~nm}$.

Until now, we obtained large quantity, uniform, and few-layer antimonene. However, it's still a question whether the nanosheets are stable as theory forecast or not, though the colloid of few-layer antimonene is stable as experiments proved. Thus, we tested the stability of few-layer antimonene in ambient condition by X-ray photoelectron spectroscopy (XPS), energy dispersive spectrum (TEM-EDX) and Raman spectrum. Figure $6 \mathrm{a}$ and $6 \mathrm{~b}$ give the XPS-peak-differenating analysis for few-layer antimonene just prepared and after 30 days, the full spectrums show in Figure S3. We can see there's no oxidation for just prepared few-layer antimonene and a slight oxidation in $\mathrm{Sb}$ nanosheets after 30 days. And this result also can be 
proved by the TEM-EDX mapping of few-layer antimonene, in the image (Figure 6c), we can see the carbon film in micro $\mathrm{Cu}$ grid through the nanosheet, which suggested the nanosheet is ultrathin. And the elemental distribution shows the content of $\mathrm{Sb}$ is more than $\mathrm{O}$, this can be accepted considering the nanosheets were drying in air. The EDX spectrum is shown in Figure S4, the mass ratio of Sb and O is 96.47/3.53.

And we also measured the thermal stability of few-layer antimonene (Figure S5). First we heated the few-layer antimonene at $100{ }^{\circ} \mathrm{C}, 200{ }^{\circ} \mathrm{C}, 300{ }^{\circ} \mathrm{C}$, and $400{ }^{\circ} \mathrm{C}$ for 30 minutes, respectively. Then we measured the XRD of the samples, it showed that few-layer antimonene were stability even heated at $200{ }^{\circ} \mathrm{C}$ after 30 minutes. And when temperature reached at $300{ }^{\circ} \mathrm{C}$, a slight oxidation occurred. And at $400^{\circ} \mathrm{C}$, the oxidation is obvious. Thus, we think the antimonene is stable at higher temperature (about $200^{\circ} \mathrm{C}$ ).

To further study the crystal structure and quality of fabricated few-layer antimonene, we measured micro-Raman spectra ${ }^{30-33}$ of typical few-layer antimonene dispersed on silicon substrate as well as the commercial antimony powder (Figure 6d). Raman peaks of few-layer antimonene match well with antimony powder, ${ }^{34}$ clearly verifying the theoretically predicted $\beta$-phase characteristic of antimonene. ${ }^{35}$ The $E_{g}$ peak at $\sim 112 \mathrm{~cm}^{-1}$ is an in-plane vibrational mode, and $A_{1 \mathrm{~g}}$ peak at $\sim 148 \mathrm{~cm}^{-1}$ is out-of-plane vibrational mode. Interestingly, the characteristic Raman peaks (Figure 6e) of few-layer antimonene were well preserved after storage in air for one month, without any additional peaks from antimony trioxide or antimony pentaoxide. ${ }^{36}$ This observation strongly demonstrates that unlike black phosphorus, few-layer antimonene is quite stable in air. 

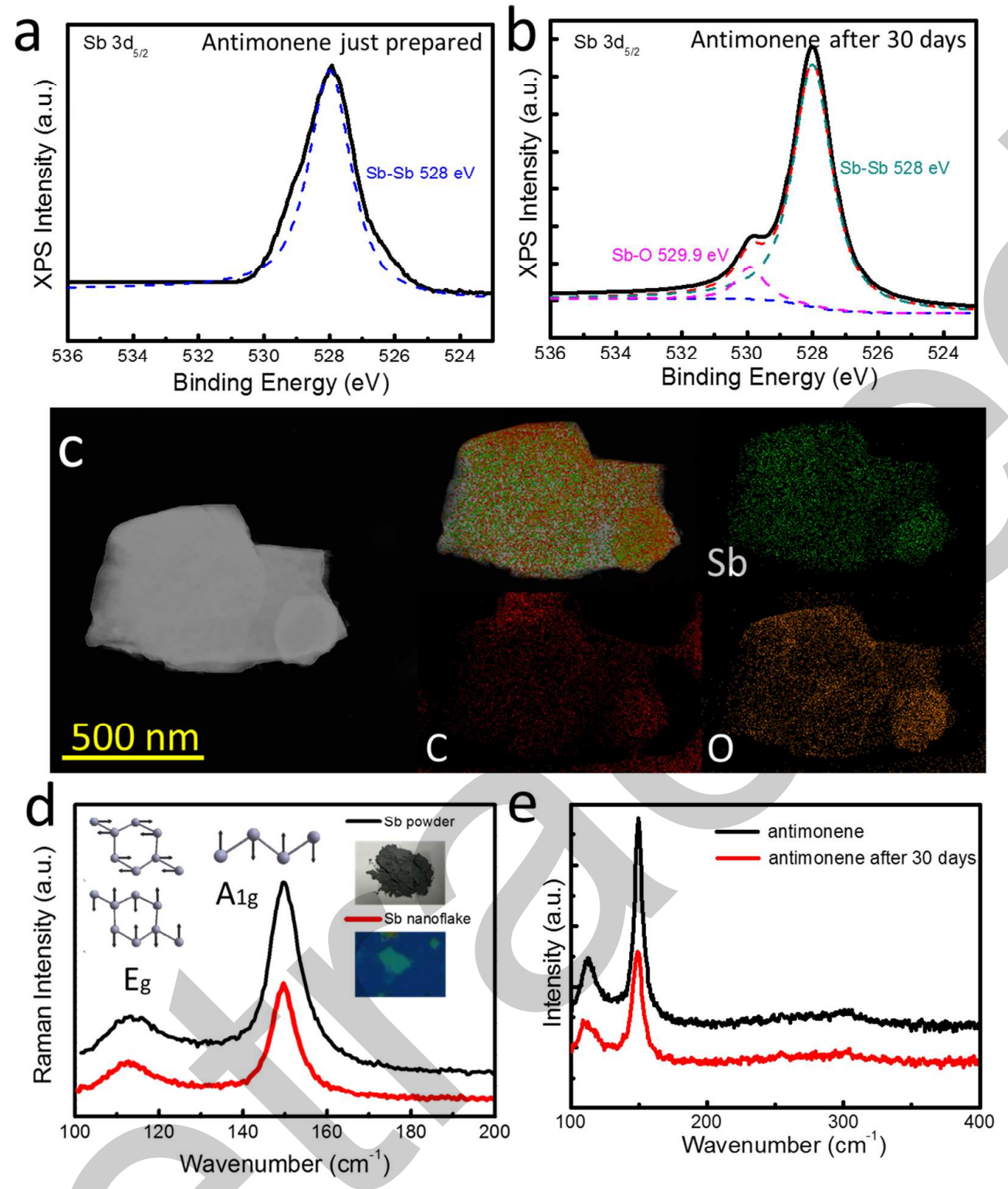

Figure 6. (a) XPS spectra of Sb $3 d_{5 / 2}$, there's no oxidiation. (b) XPS spectra $\mathrm{Sb} 3 \mathrm{~d}_{5 / 2}$, showing slight oxidation of the Sb nanosheets. (c) TEM-EDX mapping of few-layer antimonene in micro $\mathrm{Cu}$ grid. Element mapping suggests the nanosheet was slight oxidation. (d) Raman spectra of antimony powder and typical nanosheets as the right insets show. The two peaks represent two different vibrational modes respectively, which are showed in the left insets. Those two peaks are matched well with $\beta$-phase antimony. (e) Raman spectrum of few-layer antimonene just prepared and kept in air for one month. Note that the Raman spectrum remains unchanged, which shows the good stability of $\mathrm{Sb}$ nanosheets in air. 
Moreover, the evolution of few-layer antimonene Raman peaks with different thickness (Figure 7a-7f) was found to be of very high regularity (Figure $7 \mathrm{~g}$ and $7 \mathrm{~h}$ ), which could be developed as an non-destructive way to identify thickness. ${ }^{33}$ Both $\mathrm{E}_{\mathrm{g}}$ and $A_{1 \mathrm{~g}}$ peaks weaken with the decrease of thickness, calibrated by the $520 \mathrm{~cm}^{-1}$ peaks from Si substrate (Figure $7 \mathrm{~g}$ ). The plot of the intensity ratio of Si peak to few-layer antimonene $A_{1 g}$ peak versus the thickness (Figure $7 \mathrm{~h}$, the blue line) obeys exponential damping law of Formula (1), where $\mathrm{A}=\ln \left(\mathrm{I}_{\mathrm{si}} / \mathrm{I}_{\mathrm{A} 1 \mathrm{~g}}\right)$ and $\mathrm{h}$ is the thickness. In addition, the $\mathrm{E}_{\mathrm{g}}$ peak was found to weaken faster than $\mathrm{A}_{1 \mathrm{~g}}$ peak, and there is also an exponential relationship, expressed as Formula (2), where B is defined as $\ln \left(\mathrm{I}_{\mathrm{Eg}} / \mathrm{I}_{\mathrm{Alg}}\right)$. Similar phenomena have also been found in other $2 \mathrm{D}$ materials. ${ }^{33}$ One of the possible explanation is that the in-plane vibration is directly related the number of layers and the interfacial vibration has less dependence with the layers. Because the in-plane vibration can be approximately regarded independent in different layers, the intensity will be linear superposition when adding a new layer. Thus, when the thickness decreasing, the $\mathrm{E}_{\mathrm{g}}$ mode intensity will attenuate rapidly to the $\mathrm{A}_{1 \mathrm{~g}}$ mode. Obviously, the $A \sim h$ and $B \sim h$ linear relationships are very facile tools to quantitatively identify the thickness of few-layer antimonene through non-destructive Raman measurements.

$\mathrm{A}=-0.168 \mathrm{~h}+5.594$

$\mathrm{B}=0.091 \mathrm{~h}-2.846$ 

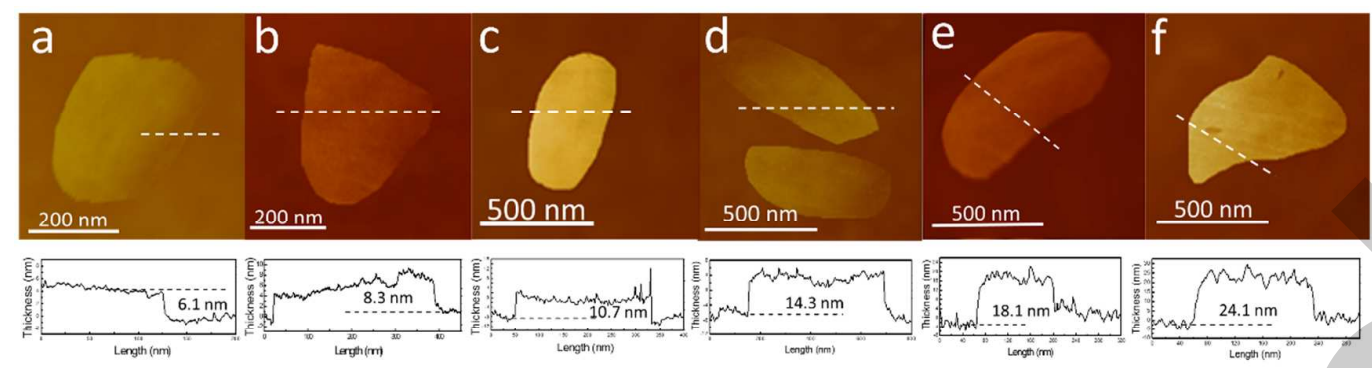

g

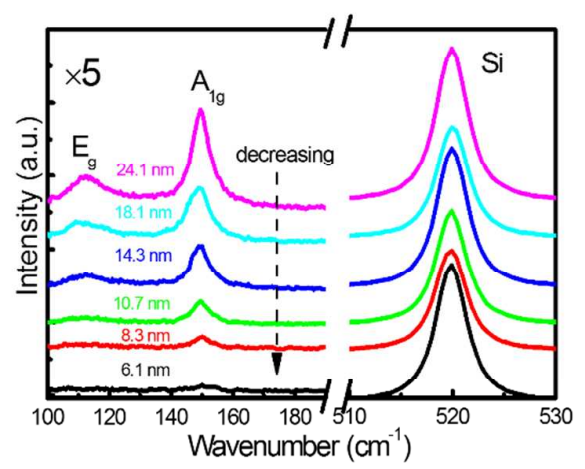

h

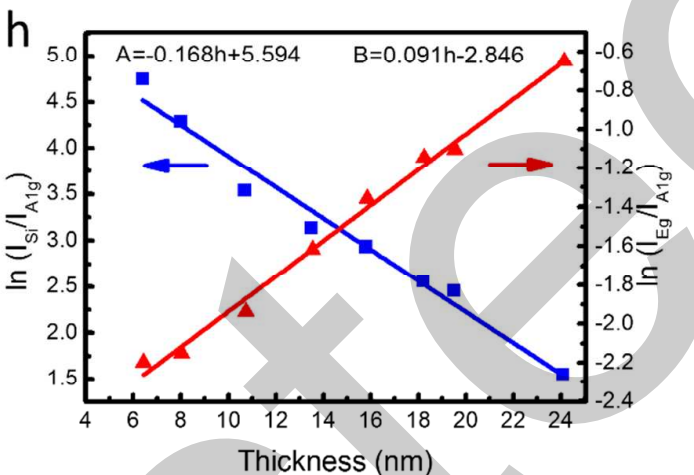

Figure 7. (a)-(f) AFM images and height analysis correlation to micro-Raman in Figure 3(g). (g) Raman spectra of several nanosheets with different thickness ranging from a few nanometers to about $40 \mathrm{~nm}$. (h) The intensity ratio of Si peak to $A_{1 g}$ peak (blue line) and the intensity ratio of $\mathrm{E}_{\mathrm{g}}$ peak to $\mathrm{A}_{1 \mathrm{~g}}$ peak (red line) versus nanosheet thickness.

Atomical Structure of Few-layer Antimonene. Atomical structure analysis is very important for researchers to identify materials' phase, constituents and predict its potential applications. Figure 8a presents the TEM image of a typical ultrathin nanosheet (the low magnification TEM image is given in Figure S6a), the percent of such kind of nanosheets can reach to $\sim 8 \%$. The edge has been rolled up by the surface tension because it is too thin, which actually is a common phenomenon observed in Figure 4c. The distance between lattice fringes, $0.214 \mathrm{~nm}$, is ascribed to the (110) plane of antimony. The FFT, pattern from the circled area is indexed along [001] zone axis and confirms the good crystallinity. The high resolution transmission electron microscope (HRTEM) (Figure 8b) shows that two hexagonal structures with different brightness nest each other. For the image, the spherical aberration coefficient was adjusted for $\mathrm{Cs}=-13 \mu \mathrm{m}$, and an overfocus of $\Delta \mathrm{Z}=+7 \mathrm{~nm}$ was used. Under this 
condition, atom columns appear bright on a dark background. Enlargement of the fold on the bilayer edge (Figure 8c) reveals a zigzag structure, which reflects the side view of few-layer antimonene (Figure 8d) because of its buckling. Moreover, we found a few nanosheets cleaving along the [010] zone axis, which gave us a chance to understand the lateral structure (Figure S7). These images also offer a direct evidence that we obtained the layered materials.

Figure 8e presents the TEM image of a thicker nanosheet (the low magnification TEM image is given in Figure S6b), which shows a flat edge with unambiguous atomic steps. The Fast Fourier Transform (FFT) of the circled area (inserted in Figure 8e) reveals that the HRTEM image was taken along the [001] zone axis. The derived lattice parameters of the crystal from FFT pattern, $a=b=4.3084$, are consistent with the lattice parameters of rhombohedral Sb. The measured HRTEM image (Figure 8f) shows that all the $\mathrm{Sb}$ atoms are closely packed into a hexagonal structure. And the image was also obtained under the same conditions of Figure 8b. 
a
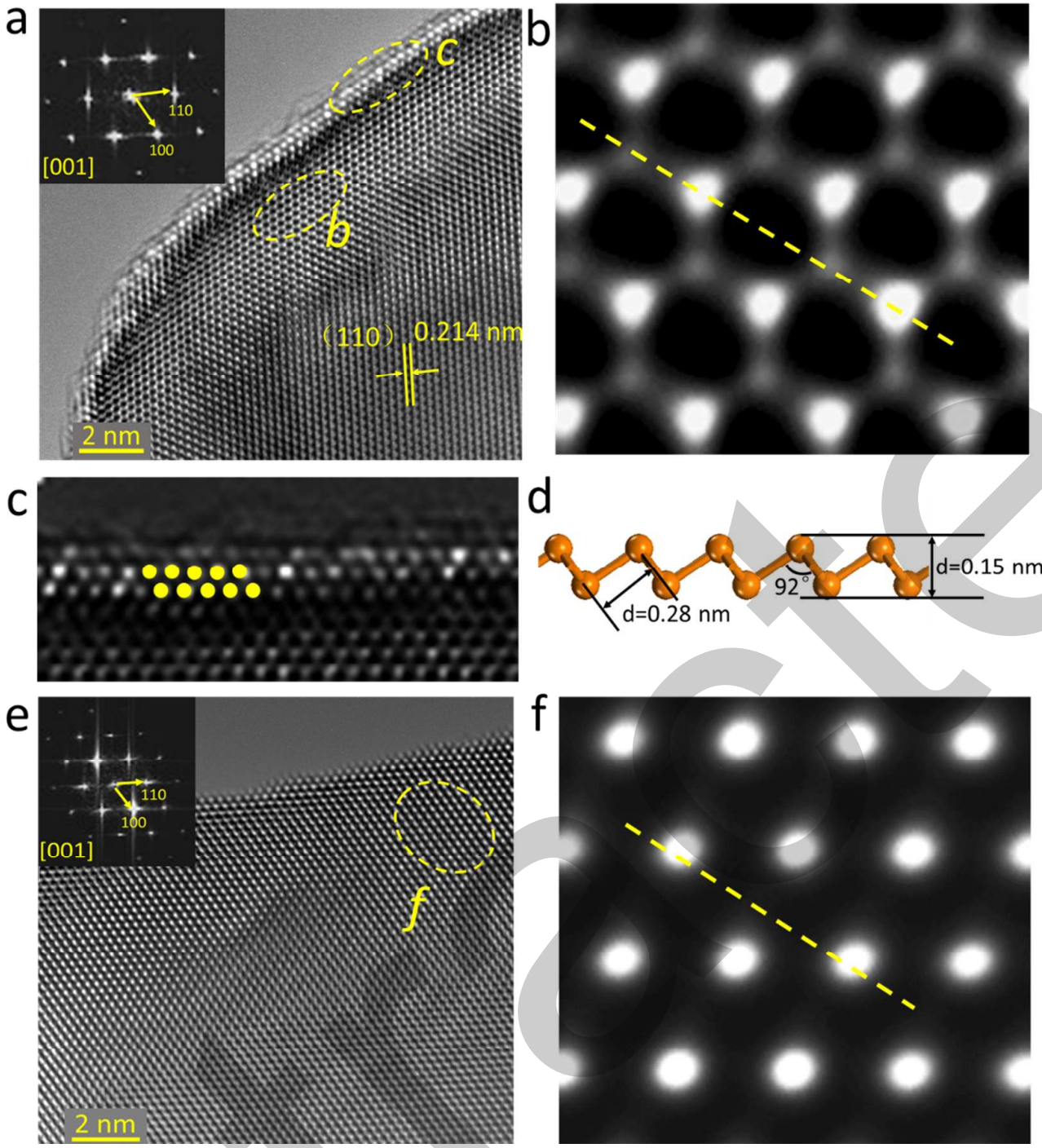

e

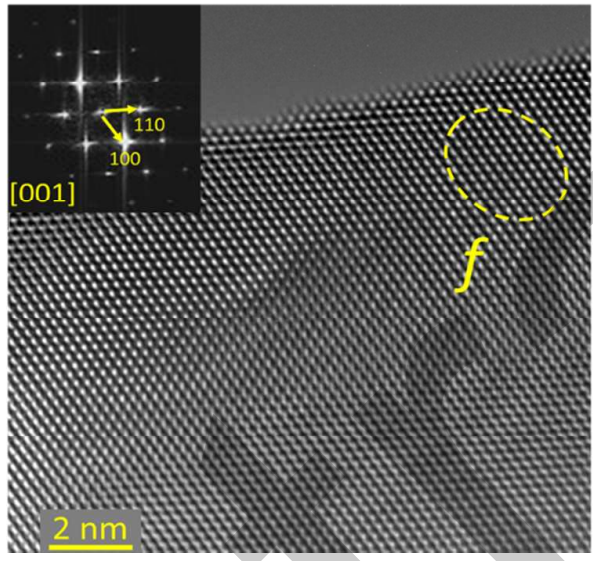

Figure 8. (a) TEM image of a typical bilayer antimonene. Inset: the FFT of the antimonene. (b) Enlarged experimentally obtained HRTEM image of bilayer antimonene. (c) The HRTEM image of few-layer antimonene edge which can reflect the structure model (d) of single layer Sb nanosheet on side view. (e) TEM image of a typical multilayer antimonene. Inset: the FFT of the nanosheets. (f) Enlarged experimentally obtained HRTEM image of multilayer antimonene.

Note that Figure $8 \mathrm{~b}$ and Figure $8 \mathrm{f}$ are obvious different, we think there may be a possible way to judge the number of layers. ${ }^{21,37}$ Thus, we give the simulated images of monolayer, bilayer, and multilayer antimonene (Figure 9a-9c). The simulated images of antimonene with different thicknesses were obtained by MactempasX 
software package, and the same microscopy parameters as in the experiments were employed. Interestingly, we found the simulated images of bilayer antimonene with $\mathrm{AB}$ stacking order and multilayer antimonene with $\mathrm{ABC}$ stacking order are similar with the HRTEM images we obtained, which suggested there may be bilayer and multilayer antimonenes in the samples we prepared. To further illustrate the consistency of the measured and simulated images, we examined the line scanning profiles along the dash line as in Figure 9d, such a quantitative analysis further proves that a bilayer antimonene was successfully prepared. Note that we also calculated the image of Sb nanosheet monolayer (Figure 9a), however, no corresponding image was observed in our experiments.
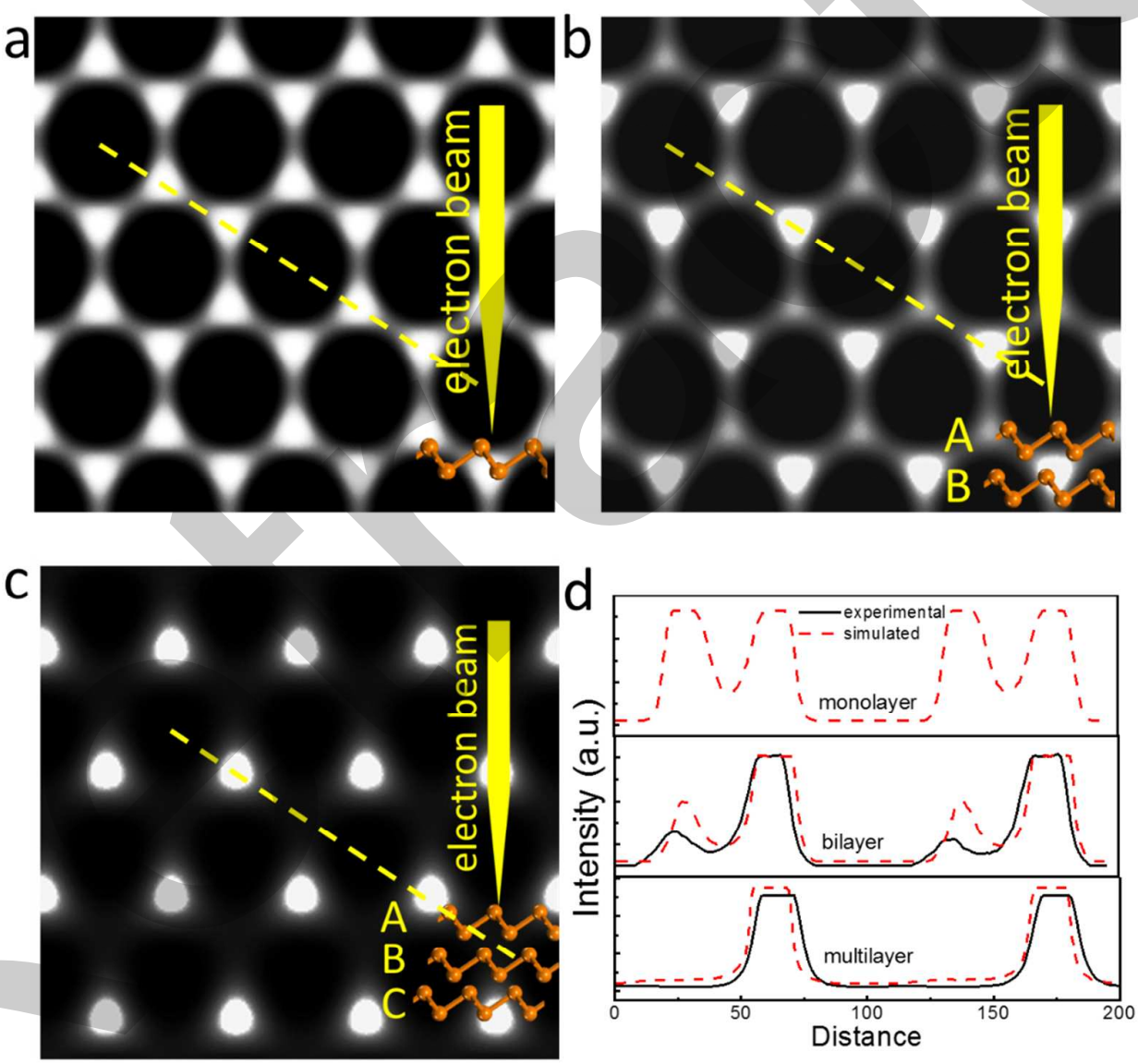

Figure 9. (a) Simulated HRTEM images of monolayer antimonene. Inset: a schematic diagram of an electron beam through the monolayer antimonene. (b) Simulated HRTEM images of bilayer antimonene. Inset: a schematic diagram of an electron beam through the AB stacking bilayer antimonene. (c) Simulated HRTEM images of 
multilayer antimonene. Inset: a schematic diagram of an electron beam through the ABC stacking multilayer antimonene. (d) The line scanning profiles taken directly from the experimental (solid line) and calculated (dash line) images.

Optical Limiting Behavior and the Mechanism of Few-layer Antimonene. To satisfy the demands from laser protective and all-optical switch technology applications, OL performance of 2D materials has become a new hotspot. Recently, giant NLO effect has been found in graphene $e^{38-39}$ and other 2D semiconductors ${ }^{40-41}$. Usually, OL refers to visible or NIR region, extending up to $1064 \mathrm{~nm}$. Though some organic dyes $^{42}$ and few-layer graphene oxide ${ }^{43}$ exhibit OL at 1600 and $1800 \mathrm{~nm}$, their applications are hindered by the very low transparency. ${ }^{44}$ Thus, to widen laser protective window and other NLO applications, it is highly desirable, also a great challenge, to develop OL material-based transparent solid glass devices with both broadband OL and high transparency. This is the general problem of all NLO materials and application. Interestingly, when dispersed in solid Ormosil matrices, the antimonene not only have a wide band transparency, but also have a good performance in optical limiting.

Figure 10a and $10 \mathrm{~b}$ show the responses of the few-layer antimonene ethanol dispersions to $532 \mathrm{~nm}$ and $1064 \mathrm{~nm}$ nanosecond lasers, compared with few-layer graphene (Figure S8) ethanol dispersions at same linear optical transmission of $70 \%$. With the increase of input laser energy, a platform emerges and the output energy keeps nearly steady after an inflection point. At high input incidence density, the slope $\mathrm{d}_{\text {Output }} / \mathrm{d}_{\text {Input }}$ gives the limiting differential transmittance $\left(\mathrm{T}_{\mathrm{c}}\right)$, indicating the output clamping characteristics of samples, ${ }^{45}$ the smaller of $\mathrm{T}_{\mathrm{c}}$, the better of OL ability. When irradiated by $532 \mathrm{~nm}$ laser, the $\mathrm{T}_{\mathrm{c}}$ value of few-layer antimonene dispersion is only $5 \%$, compared with $15 \%$ of few-layer graphene dispersion (shown in Figure 10c) at about $40 \mathrm{~J} / \mathrm{cm}^{2}$, which suggests that over $90 \%$ energy is limited when the input fluence is $40 \mathrm{~J} / \mathrm{cm}^{2}$. When irradiated by $1064 \mathrm{~nm}$ laser, though the $\mathrm{T}_{\mathrm{c}}$ of few-layer antimonene increases to $24 \%$ at $25 \mathrm{~J} / \mathrm{cm}^{2}$, it's still smaller than graphene, which increases to $28 \%$ at the same input fluence. 
By plotting the transmittance versus input energy characteristic (right panels of Figure 10a and 10b), we can obtain two other important parameters of OL properties, namely the $\mathrm{OL}$ onset threshold $\left(\mathrm{F}_{\mathrm{ON}}\right)$ and the optical limiting threshold $\left(\mathrm{F}_{\mathrm{OL}}\right.$, defined as the input fluence point at which the normalized transmittance drops to $50 \%$ ). Note that smaller $\mathrm{F}_{\mathrm{ON}}$ and $\mathrm{F}_{\mathrm{OL}}$ values indicate better $\mathrm{OL}$ ability. When irradiated by $532 \mathrm{~nm}$ laser, for the few-layer antimonene dispersions, the $\mathrm{F}_{\mathrm{ON}}$ is $0.75 \mathrm{~J} / \mathrm{cm}^{2}$ and $\mathrm{F}_{\mathrm{OL}}$ is 5.2 $\mathrm{J} / \mathrm{cm}^{2}$. Compared with graphene dispersion (the $F_{O N}$ is $0.64 \mathrm{~J} / \mathrm{cm}^{2}$ and $F_{O L}$ is $6.3 \mathrm{~J} / \mathrm{cm}^{2}$, Figure 10c), the few-layer antimonene dispersion is more sensitive to the optical energy fluence increase at this wavelength. When irradiated by $1064 \mathrm{~nm}$ laser, the $\mathrm{F}_{\mathrm{ON}}$ of antimonene dispersion is $0.72 \mathrm{~J} / \mathrm{cm}^{2}$ and $\mathrm{F}_{\mathrm{OL}}$ is $6.3 \mathrm{~J} / \mathrm{cm}^{2}$, much smaller than those of graphene, especially for the $\mathrm{F}_{\mathrm{OL}}$ (Figure 10c).
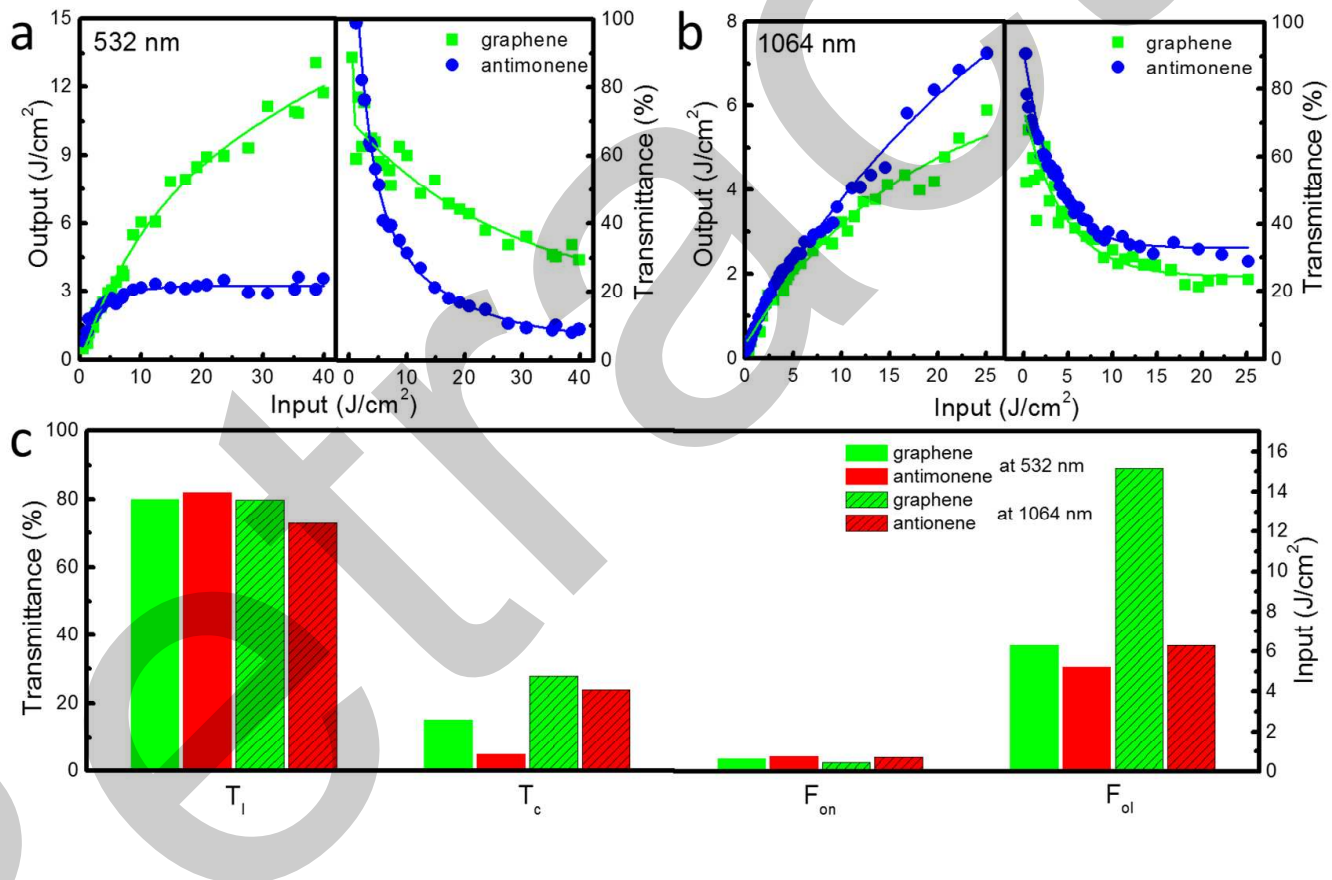

Figure 10. (a) (b) The OL properties of few-layer antimonene and few-layer graphene ethanol dispersion with same linear optical transmission of $70 \%$ at $532 \mathrm{~nm}$ and 1064 $\mathrm{nm}$ respectively. The comparison showed the OL behavior of antimonene is better than graphene at $532 \mathrm{~nm}$. (c) The comparison graphene dispersion and antimonene dispersion about their OL behaviors at $532 \mathrm{~nm}$ and $1064 \mathrm{~nm}$.

To explore the mechanism for so outstanding OL performances of few-layer 
antimonene, we performed open-aperture and close-aperture nanosecond Z-scan measurements under laser energy of $8 \mu \mathrm{J}$ (Figure 11a and 11b). The open-aperture Z-scan curve in Figure 11a exhibits two-photon absorption characteristics, which are fitted with various two photon absorption processes by using analytical methods, and gives a nonlinear optical absorption coefficient $\left(\beta_{\mathrm{oa}}\right)$ of about $138 \mathrm{~cm} / \mathrm{GW}$ at $532 \mathrm{~nm}$, which is larger than most $2 \mathrm{D}$ materials. Thus, the generated principle of nonlinear optical absorption behavior of few-layer antimonenes conforms to the two photon absorption. ${ }^{46-49}$ On the other hand, the close-aperture nanosecond Z-scan in Figure $11 \mathrm{~b}$ gives positive nonlinear refraction index values related to self-focusing effect. Considering the OL performance maybe come from the dispersion system rather than antimonene itself, we measured the responses of the few-layer antimonene in different solvents such as 1,2-dichlorobenzene (DCB), ethanol, deionized water (DI), dimethyl formamide (DMF), and N-methyl-2-pyrrolidone (NMP), to $532 \mathrm{~nm}$ nanosecond lasers. The results (Figure 11c) shows there's no solvent effect for few-layer antimonene dispersion. Thus, the solvent microbubble mechanism ${ }^{50}$ can be excluded, which plays an important role in the nonlinear scattering process. ${ }^{38}$ Furthermore, when we deviated detector in $5^{\circ}$ range to capture scattering signals, the scattering peaks exhibited well symmetry in open aperture Z-scan measurement till the laser energy reached as high as $140 \mu \mathrm{J}$ (Figure 11d), which confirms there's no a strong nonlinear scattering contribution. Thus, all these results suggest that the outstanding OL of antimonene is mainly originated from nonlinear absorption, but also contributed together by nonlinear refraction. 

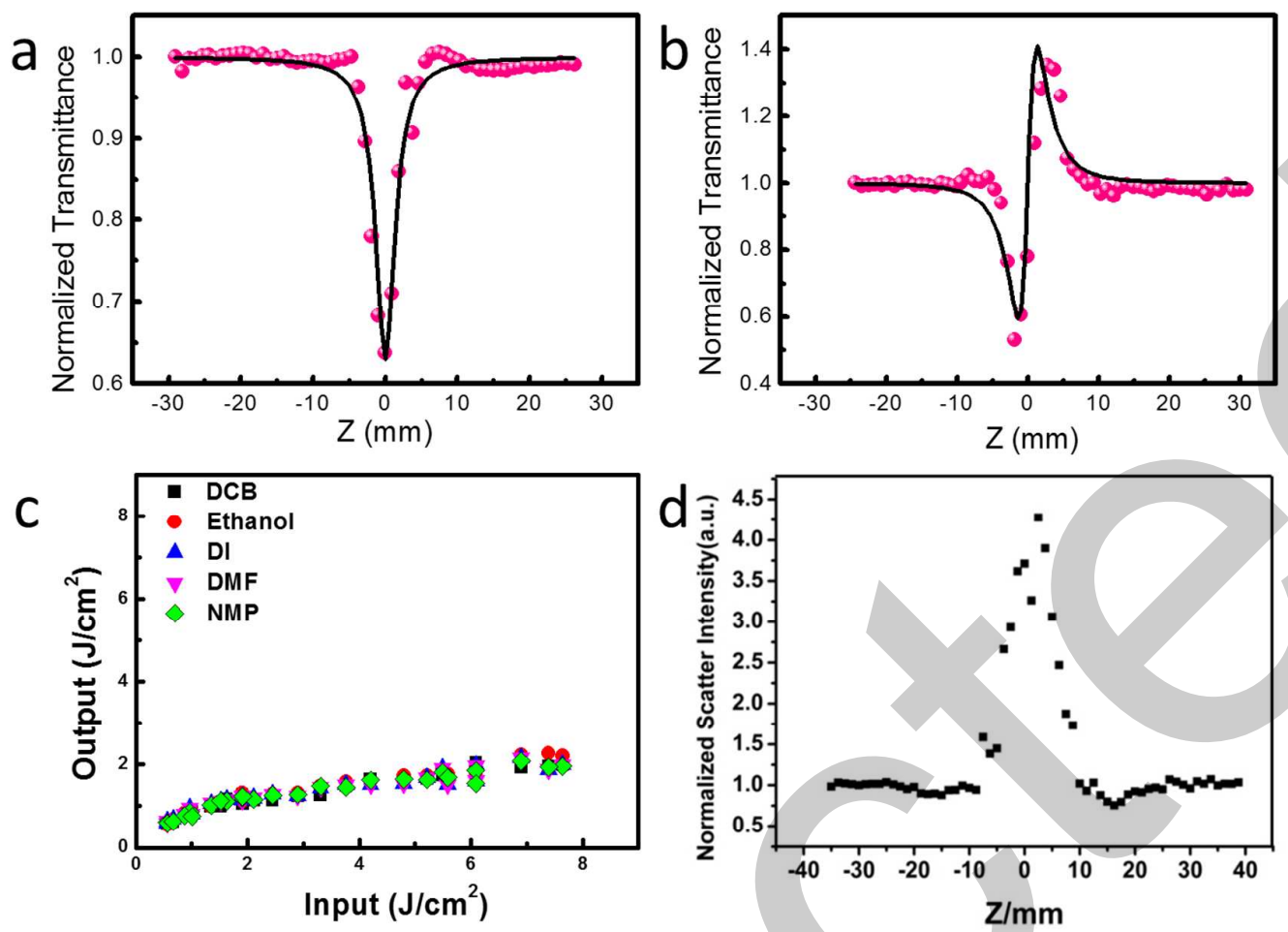

Figure 11. (a) Open-aperture Z-scan results of few-layer antimonene dispersion at $532 \mathrm{~nm}$. (b) Close-aperture Z-scan results of few-layer antimonene dispersion at $532 \mathrm{~nm}$. (c) The optical limiting effects of few-layer antimonene dispersions in different solvents. These data suggest that the optical limiting effects have no differences in different solvents of few-layer antimonene. (d) Nonlinear scattering results of few-layer antimonene dispersions. The detector was set at $5^{\circ}$ to the direction of the laser beam to collect the nonlinear scattering signal at the open aperture Z-scan studies.

Applications of Few-layer Antimonene in Laser Protection. To verify the practicability of above excellent OL properties, the solid Ormosil gel glass monoliths doped with few-layer antimonene (after polished) have been fabricated. Figure 12a gives a schematic diagram about the preparation process ${ }^{51}$, and the details were given in the Method. Figure 12b and Figure S9 show the solid Ormosil gel glass, which are smooth as Figure 12c shows. The dispersion of few-layer antimonene inside glasses is very uniform as confirmed by the energy disperse spectrum (EDS) mapping and SEM 
image on the fractured section surface of sol-gel glass (Figure 5d and Figure S10). Note that the loading fractions of graphene in transparent composites are often less than $0.1 \%$ and the low transparency at high doping level often limits their OL applications (Figure S9). Encouragingly, few-layer antimonene can be doped into Ormosil glass in large quantity, even with concentration up to $1 \mathrm{wt} \%$, which is much higher than graphene and other optical limiting materials, and the glass is still transparent enough in the visible waveband. The transmission spectra (Figure S11) clearly exhibit that all the few-layer antimonene doped Ormosil glasses have good transparency in the visible and NIR region, with a transmittance $(\mathrm{T})$ of over $90 \%$ at wavelengths of $350 \sim 1350 \mathrm{~nm}$ for $0.01 \mathrm{wt} \%$ few-layer antimonene glasses and $80 \%$ for $0.1 \mathrm{wt} \%$ few-layer antimonene doped gel glass.

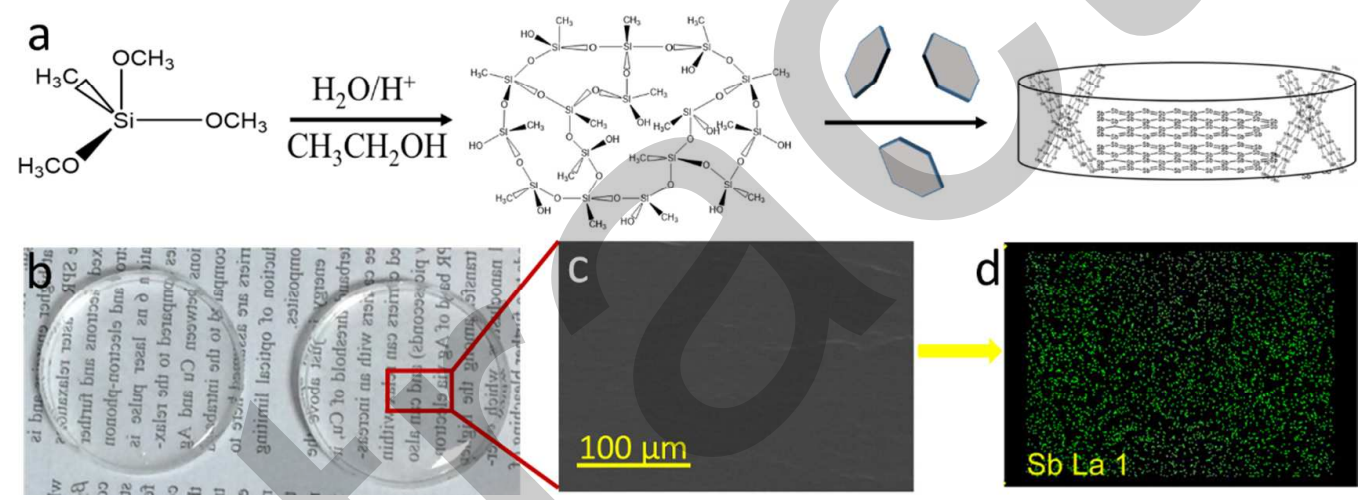

Figure 12. (a) The schematic diagrams of preparation of few-layer antimonene doped hybrid Ormosil gel glass (b) Optical photograph of polished hybrid Ormosil gel glass monoliths doped with $0.01 \mathrm{wt} \%$, and $1 \mathrm{wt} \%$ few-layer antimonene. The gel glasses show a good transmission of visible light even doped in high concentration. (c) The SEM image of the fractured section surface of hybrid Ormosil gel glass. (d) The SEM-EDS of hybrid Ormosil gel glass, few-layer antimonene dispersed uniformly in the gel glasses as images show. 

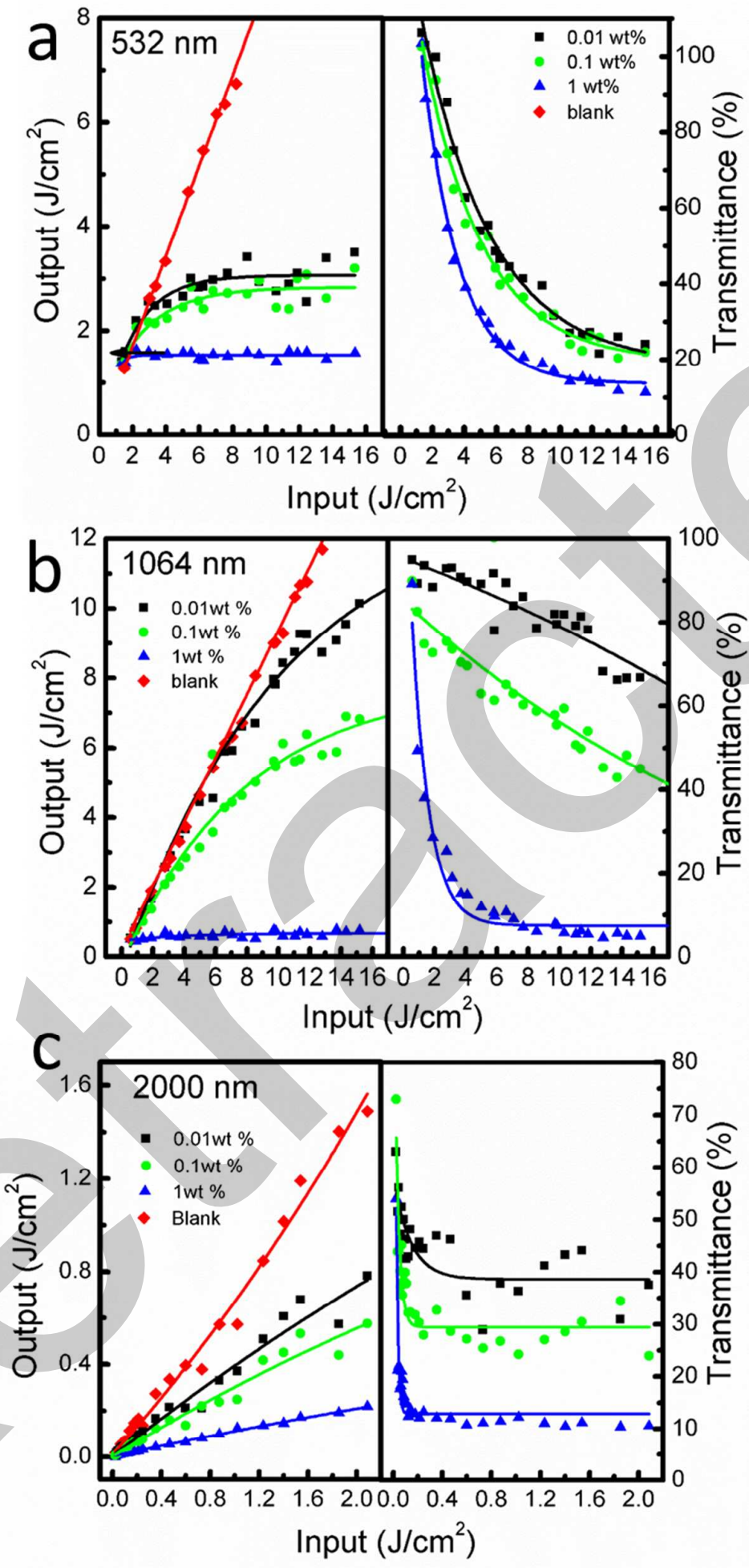

Figure 13. (a)-(c) The OL properties of gel glass with different load fractions few-layer antimonene at $532 \mathrm{~nm}, 1064 \mathrm{~nm}$ and $2000 \mathrm{~nm}$, respectively. 
OL responses of few-layer antimonene glasses to 532, 1064 and $2000 \mathrm{~nm}$ lasers are plotted in Figure 13a-13c, respectively. The output fluence of the pure methyltriethoxysilane (MTES) glass shows linear dependence on input fluence, which is contrary to the nonlinear dependence after few-layer antimonene incorporation. As input fluence increase, the OL effect becomes significant. For $1 \mathrm{wt} \%$ gel glass, the $\mathrm{F}_{\text {on }}$ is $1.33 \mathrm{~J} / \mathrm{cm}^{2}, 0.56 \mathrm{~J} / \mathrm{cm}^{2}$ and $0.025 \mathrm{~J} / \mathrm{cm}^{2}$ at $532 \mathrm{~nm}, 1064 \mathrm{~nm}$ and $2000 \mathrm{~nm}$ respectively. At high input fluence, Tc reflects output clamping. The $\mathrm{T}_{\mathrm{c}}$ of $1 \mathrm{wt} \%$ gel glasses is less than $5 \%$ at both $532 \mathrm{~nm}$ and $1064 \mathrm{~nm}$, which is very practical in laser protection. Naturally, the $1 \mathrm{wt} \%$ gel glasses with the highest doping concentration exhibits the best OL properties.

And we also measured the OL responses of few-layer graphene doped glasses which have the same linear transmittance with few-layer antimonene doped glasses at $532 \mathrm{~nm}$ and $1064 \mathrm{~nm}$. The optical limiting responses of samples were shown in Figure $\mathrm{S} 12$, and the antimonene solid hybrid glasses have stronger optical limiting responses than the graphene, which was consistent with the trend of the liquid dispersion of the few-layer graphene and few-layer antimonene.

In order to apply the glasses, the stability of them is an important factor. In order to investigate the stability of antimonene in the Ormosil glasses under the strong laser irradiation, two series of experiments have been carried out. On the one hand, the Ormosil glasses doped with few-layer antimonene were placed in the optical limiting measurement system to find the energy density of damage threshold under 532 and $1064 \mathrm{~nm}$ nanosecond pulse laser. The damage thresholds of the samples at 532 and $1064 \mathrm{~nm}$ wavelengths were 22 and $12 \mathrm{~J} / \mathrm{cm}^{-2}$ displayed in Figure S13(a) and (b), respectively. The damage threshold was almost consistent with the value taken place in the manuscript because the test spot was changed at random. On the other hand, the laser power was set at 503,1016 , and $2048 \mu \mathrm{W}$, equal to $3.88,7.80$ and $15.79 \mathrm{~J} / \mathrm{cm}^{-2}$ at $532 \mathrm{~nm}$ wavelength when the laser was changed into $1064 \mathrm{~nm}$ wavelength 525, 1013 and $2003 \mu \mathrm{W}$, equal to $2.57,4.96$ and $9.81 \mathrm{~J} / \mathrm{cm}^{-2}$. Then the sample was set at the focus local of the optical lens and 100 laser pulses were recorded and the curves of normalized transmittance (NT) versus number of laser pulses were shown in Figure 
S13 (c) and (d). It can be seen that NT fluctuated slightly at a constant value and the result was consistent with the optical limiting results, in which NT decreased with the power increase. Moreover, there was no damage for each irradiated sample. All the two series of experiments confirmed that the antimonene in the Ormosil matrices were very stable and the hybrid glasses are proper for optical limiting applications.

Thus, few-layer antimonene can work well both in solutions and solid Ormosil matrices. The loading fraction of few-layer antimonene in the matrix could be easily controlled from $0 \%$ to $1 \%$ by changing the ratio of the few-layer antimonene. Properties of the Ormosil glasses could be modulated accordingly, and comparable or surpassable to those in solution. The solid Ormosil glasses can be easily gelated, cut, and polished into different geometries and sizes depending on the target design and application, and have long-term optical, thermal, and mechanical stabilities as well as high transmission and NLO \& OL performance in the visible and NIR region (532 $\mathrm{nm}-2000 \mathrm{~nm})$.

\section{Conclusions}

In summary, we prepared a large quantity, high quality few-layer antimonene via liquid exfoliation, and demonstrated their exact atomical structure and outstanding nonlinear optical properties. XRD, Raman spectroscopy and HRTEM characterizations revealed that few-layer antimonenes have layered atomic structure consisting of buckled hexagonal rings, as in the $\beta$-phase bulk antimony. Our experiments reveal that few-layer antimonene are very stable in ambient conditions as predicted by theoretical computations. Raman spectroscopy also provides a nondestructive, frequent method in identifying the thickness of few-layer antimonene. Surprisingly, few-layer antimonene present excellent $532 \mathrm{~nm} \sim 2000 \mathrm{~nm}$ broadband optical limiting and high transmission performance when dispersed in solutions or high concentration doped in Ormosil gel glasses, which might lead to many promising applications in NLO fields such as laser protection. This work demonstrates that Group 15 2D materials beyond BP could be not only a new 2D crystal family with stability in ambient conditions, but also of unique properties and applications. 


\section{Experimental Section}

Fabrication of few-layer antimonene. Few-layer antimonenes were prepared by sonication liquid phase exfoliation, in which the power and time of sonication, as well as the centrifugation speed and time are important parameters. We tested a series of experimental conditions to determine the optimized parameters. First, $200 \mathrm{mg}$ commercial antimony powders were added into $80 \mathrm{~mL}$ ethanol solvent. Then the mixture was sonicated for five hours at the power of $200 \mathrm{~W}$. The resultant dispersion was centrifuged for 20 minutes at speed of $3000 \mathrm{rpm}$. The supernatant containing antimonene was decanted gently.

Preparation of antimonenes doped Ormosil gel glasses. The materials were prepared by the hydrolysis and polycondensation of MTES in acidic medium $\left(\mathrm{H}_{2} \mathrm{O}\right.$, acetic acid or $\mathrm{HCl}, \mathrm{pH}=2.5$ ) using a modified method reported in the literature. [31] The molar ratio of MTES: ethanol: distilled water in the precursor was 3:1:3. The mixture was stirred overnight and evaporated to the initial volume of the MTES precursor. The mixture was stirred for 5-7 days. The dispersion of antimonene was added to the sol. After aging was finished, the gels were divided into several volume parts, casted into polypropylene cells individually, and dried 1-2 weeks at room temperature to give the final monoliths.

Characterization of few-layer antimonene. UV/Vis absorption spectra were obtained on a Shimadzu 3600 spectrophotometer. X-ray diffraction (XRD) patterns were recorded on a Bruker D8 multipurpose XRD system. SEM images were obtained by FEI Quanta 250FEG. AFM measurements were carried on a Bruker Multimode 8 AFM system. Raman spectrum obtained by Aramis made by HORIBA JOBIN YVON at $532 \mathrm{~nm}$. UV-Vis-near IR spectra were measured on Varian Cary 5000 UV-visible-near infrared absorption spectrophotometer. The antimonene dispersion was dropped onto a micro $\mathrm{Cu}$ grid, and toluene was allowed to evaporate at room 
temperature. TEM and HRTEM images were taken on a FEI Titan 80-300 Cs-corrected electron microscope at Nanjing University of Science and Technology. It is equipped with a spherical aberration corrector under the objective lens. By the Young's fringe technique, this instrument offers, with respect to its transfer function, an information limit better than $0.09 \mathrm{~nm}$, corresponding to $11.1 \mathrm{~nm}^{-1}$ in reciprocal space.

Optical limiting and nonlinear optical measurements. The optical limiting properties were investigated using $8 \mathrm{~ns}$ laser pulses from a $\mathrm{Nd}$ :YAG laser (Spectra-Physics Quanta-Ray INDI Pulsed Nd:YAG Laser, optical parametric oscillator) at a repetition rate of $10 \mathrm{~Hz}$, and at wavelengths of 532, 1064, $2000 \mathrm{~nm}$. The pulse energies in front of and behind the sample were monitored by energy detectors D1 and D2 (Ophir Optronics Inc. PE25). All measurements were conducted at room temperature. The solution was contained in $5 \mathrm{~mm}$ thick quartz cells, while the solid glass was fixed vertically using a clamp. The third order nonlinear optical properties of the nanosheet in solution and its gel glass were studied using Z-scan technique with $\mathrm{Nd}$ :YAG laser as light source (the pulse width of $5 \mathrm{~ns}$ and wavelength of $532 \mathrm{~nm}$ ). Simultaneously, a focusing lens setup was arranged at $5^{\circ}$ to the direct of the incident beam to monitor the scattered light from dispersions. All the Z-scan curves of liquid samples were obtained with $1 \mathrm{~mm}$ quartz cell.

Conflict of Interest: The authors declare no competing financial interest.

\section{Acknowledgements}

C. X. Huo, X. M. Sun, Z. Yan and X. F. Song contributed equally to this work. We are thankful to Junkai Ren and Shixin Liu for their help in taking the optical limiting measurements. We also thanks Professor Zhongfang Chen and his student Jinxing Gu (Department of Chemistry, Institute for Functional Nanomaterials, University of Puerto Rico, San Juan, PR 00931, USA) for their work in polishing our manuscript. This work was financially supported by the National Basic Research Program of China (2014CB931702), NSFC (51572128), NSFC-RGC (5151101197), the Fundamental Research Funds for the Central Universities, and PAPD of Jiangsu Higher Education Institutions. 
Supporting Information Available: This material is available free of charge via the Internet at http://pubs.acs.org.

\section{References}

1. Li, L.; Yu, Y.; Ye, G. J.; Ge, Q.; Ou, X.; Wu, H.; Feng, D.; Chen, X. H.; Zhang, Y., Nature nanotechnology 2014, 9, 372-377.

2. Tran, V.; Soklaski, R.; Liang, Y.; Yang, L., Physical Review B 2014, 89, 235319.

3. Ling, X.; Wang, H.; Huang, S.; Xia, F.; Dresselhaus, M. S., Proceedings of the National Academy of Sciences 2015, 112, 4523-4530.

4. Zhang, X.; Xie, H.; Liu, Z.; Tan, C.; Luo, Z.; Li, H.; Lin, J.; Sun, L.; Chen, W.; Xu, Z.; Xie, L.; Huang, W.; Zhang, H., Angewandte Chemie International Edition 2015, 54, 3653-3657.

5. Xia, F.; Wang, H.; Jia, Y., Nature Communications 2014, 5, 4458.

6. Qiao, J.; Kong, X.; Hu, Z.-X.; Yang, F.; Ji, W., Nature Communications 2014, 5, 4475.

7. Castellanos-Gomez, A.; Vicarelli, L.; Prada, E.; Island, J. O.; Narasimha-Acharya, K.; Blanter, S. I.; Groenendijk, D. J.; Buscema, M.; Steele, G. A.; Alvarez, J., 2 D Materials 2014, 1, 025001.

8. Tang, Q.; Zhou, Z.; Chen, Z., Wiley Interdisciplinary Reviews: Computational Molecular Science 2015, 5, 360-379.

9. Jing, Y.; Zhang, X.; Zhou, Z., Wiley Interdisciplinary Reviews: Computational Molecular Science 2016, 6, 5-19.

10. Zhang, S.; Yan, Z.; Li, Y.; Chen, Z.; Zeng, H., Angewandte Chemie International Edition 2015, 54, 3112-3115.

11. Kamal, C.; Ezawa, M., Physical Review B 2014, 91, 849-855.

12. Aktürk, O. Ü.; Ãzçelik, V. O.; Ciraci, S., Physical Review B 2015, 91.

13. Zhu, Z.; Guan, J.; Tománek, D., Physical Review B 2015, 91, 161404.

14. Zhang, S.; Xie, M.; Li, F.; Yan, Z.; Li, Y.; Kan, E.; Liu, W.; Chen, Z.; Zeng, H., Angewandte Chemie 2016, 128, 1698-1701.

15. Gupta, S. K.; Sonvane, Y.; Wang, G.; Pandey, R., Chemical Physics Letters 2015, 641, 169-172.

16. Zhang, Q.; Schwingenschlögl, U., Physical Review B 2016, 93, 045312.

17. Singh, D.; Gupta, S. K.; Sonvane, Y.; Lukacevic, I., Journal of Materials Chemistry C 2016, 4, 6386-6390.

18. Xu, Y.; Peng, B.; Zhang, H.; Shao, H.; Zhang, R.; Lu, H.; Zhang, D. W.; Zhu, H., arXiv preprint arXiv:1604.03422 2016.

19. Tsai, H.-S.; Chen, C.-W.; Hsiao, C.-H.; Ouyang, H.; Liang, J.-H., Chemical Communications 2016, 52, 8409-8412.

20. Lei, T.; Liu, C.; Zhao, J.-L.; Li, J.-M.; Li, Y.-P.; Wang, J.-O.; Wu, R.; Qian, H.-J.; Wang, H.-Q.; Ibrahim, K., Journal of Applied Physics 2016, 119, 015302.

21. Coleman, J. N.; Lotya, M.; O’Neill, A.; Bergin, S. D.; King, P. J.; Khan, U.; Young, K.; Gaucher, A.; De, S.; Smith, R. J., Science 2011, 331, 568-571.

22. Hernandez, Y.; Nicolosi, V.; Lotya, M.; Blighe, F. M.; Sun, Z.; De, S.; McGovern, I.; Holland, B.; Byrne, M.; Gun'Ko, Y. K., Nature nanotechnology 2008, 3, 563-568. 
23. Nicolosi, V.; Chhowalla, M.; Kanatzidis, M. G.; Strano, M. S.; Coleman, J. N., Science 2013, 340, 1226419.

24. Hanlon, D.; Backes, C.; Doherty, E.; Cucinotta, C. S.; Berner, N. C.; Boland, C.; Lee, K.; Harvey, A.; Lynch, P.; Gholamvand, Z.; Zhang, S.; Wang, K.; Moynihan, G.; Pokle, A.; Ramasse, Q. M.; McEvoy, N.; Blau, W. J.; Wang, J.; Abellan, G.; Hauke, F.; Hirsch, A.; Sanvito, S.; O'Regan, D. D.; Duesberg, G. S.; Nicolosi, V.; Coleman, J. N., Nat Commun 2015, 6, 8563.

25. Huo, C.; Yan, Z.; Song, X.; Zeng, H., Science Bulletin 2015, 60, 1994-2008.

26. Zeng, Z.; Sun, T.; Zhu, J.; Huang, X.; Yin, Z.; Lu, G.; Fan, Z.; Yan, Q.; Hng, H. H.; Zhang, H., Angewandte Chemie International Edition 2012, 51, 9052-9056.

27. Zeng, Z.; Yin, Z.; Huang, X.; Li, H.; He, Q.; Lu, G.; Boey, F.; Zhang, H., Angewandte Chemie International Edition 2011, 50, 11093-11097.

28. Yasaei, P.; Kumar, B.; Foroozan, T.; Wang, C.; Asadi, M.; Tuschel, D.; Indacochea, J. E.; Klie, R. F.; Salehi-Khojin, A., Advanced Materials 2015, 27, 1887-1892.

29. Shih, C.-J.; Vijayaraghavan, A.; Krishnan, R.; Sharma, R.; Han, J.-H.; Ham, M.-H.; Jin, Z.; Lin, S.; Paulus, G. L. C.; Reuel, N. F.; Wang, Q. H.; Blankschtein, D.; Strano, M. S., Nat Nano 2011, 6, 439-445.

30. Ferrari, A.; Meyer, J.; Scardaci, V.; Casiraghi, C.; Lazzeri, M.; Mauri, F.; Piscanec, S.; Jiang, D.; Novoselov, K.; Roth, S., Physical review letters 2006, 97, 187401.

31. Calizo, I.; Balandin, A.; Bao, W.; Miao, F.; Lau, C., Nano letters 2007, 7, 2645-2649.

32. Yan, Z.; Jiang, C.; Pope, T.; Tsang, C.; Stickney, J.; Goli, P.; Renteria, J.; Salguero, T.; Balandin, A., Journal of Applied Physics 2013, 114, 204301.

33. Hajiyev, P.; Cong, C.; Qiu, C.; Yu, T., Scientific Reports 2013, 3, 2593.

34. Lannin, J.; Calleja, J.; Cardona, M., Physical Review B 1975, 12, 585.

35. Wang, G.; Pandey, R.; Karna, S. P., ACS Applied Materials \& Interfaces 2015, 7, 11490-11496.

36. Mestl, G.; Ruiz, P.; Delmon, B.; Knozinger, H., The Journal of Physical Chemistry 1994, 98, 11276-11282.

37. Khan, U.; O'Neill, A.; Lotya, M.; De, S.; Coleman, J. N., Small 2010, 6, 864-871.

38. Lim, G.-K.; Chen, Z.-L.; Clark, J.; Goh, R. G.; Ng, W.-H.; Tan, H.-W.; Friend, R. H.; Ho, P. K.; Chua, L.-L., Nature photonics 2011, 5, 554-560.

39. Tao, L.; Zhou, B.; Bai, G.; Wang, Y.; Yu, S. F.; Lau, S. P.; Tsang, Y. H.; Yao, J.; Xu, D., The Journal of Physical Chemistry C 2013, 117, 23108-23116.

40. Dong, N.; Li, Y.; Feng, Y.; Zhang, S.; Zhang, X.; Chang, C.; Fan, J.; Zhang, L.; Wang, J., Scientific Reports 2015, 5, 14646.

41. Jie, W.; Chen, X.; Li, D.; Xie, L.; Hui, Y. Y.; Lau, S. P.; Cui, X.; Hao, J., Angewandte Chemie 2015, 54, 1201-1205.

42. Chateau, D.; Bellier, Q.; Chaput, F.; Feneyrou, P.; Berginc, G.; Maury, O.; Andraud, C.; Parola, S., Journal of Materials Chemistry C 2014, 2, 5105-5110.

43. Liaros, N.; Koudoumas, E.; Couris, S., Applied Physics Letters 2014, 104, 191112.

44. Zheng, X.; Feng, M.; Zhan, H., Journal of Materials Chemistry C 2013, 1, 6759-6766.

45. Lili, T.; Hui, L.; Bo, Z.; Siu Fung, Y.; Ping, L. S.; Yang, C.; Kin Hung, F.; Yuen Hong, T.; Jianquan, Y.; Degang, X., Nanoscale 2014, 6, 9713-9.

46. Sheik-Bahae, M.; Said, A. A.; Wei, T.-H.; Hagan, D. J.; Van Stryland, E. W., Quantum Electronics, IEEE Journal of 1990, 26, 760-769.

47. Boggess Jr, T. F.; Bohnert, K. M.; Mansour, K.; Moss, S. C.; Boyd, I. W.; Smirl, A. L., Quantum Electronics, IEEE Journal of 1986, 22, 360-368. 
48. He, J.; Qu, Y.; Li, H.; Mi, J.; Ji, W., Optics express 2005, 13, 9235-9247.

49. Kumbhakar, P.; Kole, A. K.; Tiwary, C. S.; Biswas, S.; Vinod, S.; Taha - Tijerina, J.; Chatterjee, U.; Ajayan, P. M., Advanced Optical Materials 2015, 3, 828-835.

50. Wang, J.; Hernandez, Y.; Lotya, M.; Coleman, J. N.; Blau, W. J., Advanced Materials 2009, 21, 2430-2435.

51. Xie, Z.; Wang, F.; Liu, C. Y., Adv Mater 2012, 24, 1716-21. 


\section{TOC Figure}

High yield, ultrathin antimonene are prepared by liquid exfoliation. Their atomic level structure is elucidated. And ultrathin antimonenes show outstanding optical limiting performance in the visible and near infrared ray (NIR) region and high transmission when dispersed in solutions or high concentration doped in Ormosil gel glasses, which might lead to many promising applications in nonlinear optical (NLO) fields such as laser protection.
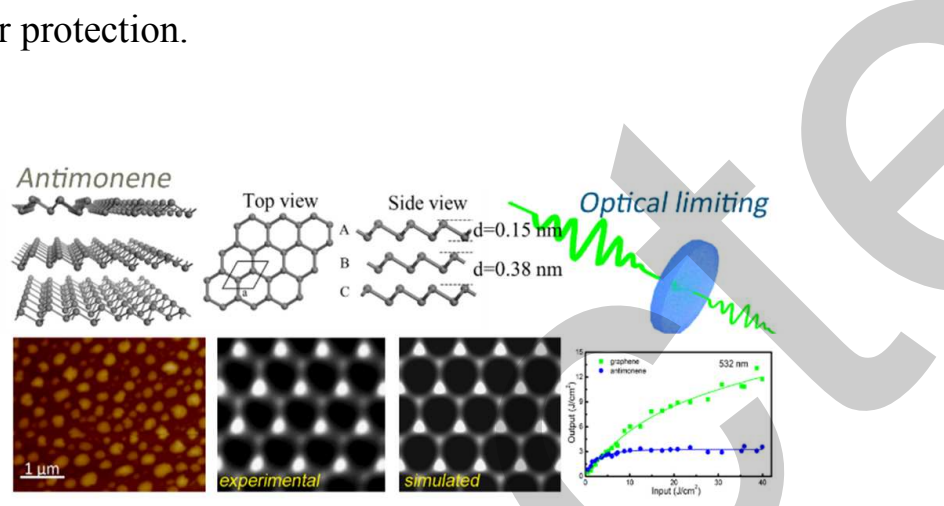